\title{
The impact of Rhodiola rosea on the gut microbial community of Drosophila melanogaster
}

\author{
Khachik E. Labachyan (D, Dara Kiani, Evgueni A. Sevrioukov, Samuel E. Schriner and Mahtab Jafari* (D)
}

\begin{abstract}
Background: The root extract of Rhodiola rosea has historically been used in Europe and Asia as an adaptogen, and similar to ginseng and Shisandra, shown to display numerous health benefits in humans, such as decreasing fatigue and anxiety while improving mood, memory, and stamina. A similar extract in the Rhodiola family, Rhodiola crenulata, has previously been shown to confer positive effects on the gut homeostasis of the fruit fly, Drosophila melanogaster. Although, $R$. rosea has been shown to extend lifespan of many organisms such as fruit flies, worms and yeast, its antiaging mechanism remains uncertain. Using D. melanogaster as our model system, the purpose of this work was to examine whether the anti-aging properties of $R$. rosea are due to its impact on the microbial composition of the fly gut.
\end{abstract}

Results: Rhodiola rosea treatment significantly increased the abundance of Acetobacter, while subsequently decreasing the abundance of Lactobacillales of the fly gut at 10 and 40 days of age. Additionally, supplementation of the extract decreased the total culturable bacterial load of the fly gut, while increasing the overall quantifiable bacterial load. The extract did not display any antimicrobial activity when disk diffusion tests were performed on bacteria belonging to Microbacterium, Bacillus, and Lactococcus.

Conclusions: Under standard and conventional rearing conditions, supplementation of $R$. rosea significantly alters the microbial community of the fly gut, but without any general antibacterial activity. Further studies should investigate whether $R$. rosea impacts the gut immunity across multiple animal models and ages.

Keywords: Drosophila melanogaster, Rhodiola rosea, Acetobacter pomorum, Lactobacillus plantarum, Bacterial load, Herbal extracts, Colony forming units, Quantitative RT-PCR, Disk diffusion, 16S rRNA gene sequencing

\section{Background}

Rhodiola rosea, informally referred to as the 'golden root' or 'arctic root', is an adaptogenic plant that has been reported to display positive effects on central nervous system activity and cardiovascular function [1-4]. The additional therapeutic effects of $R$. rosea, which derive primarily from its root extract, have been outlined in clinical trials for improving mental and physical work capacity during stress, alleviating mental distress, and ameliorating symptoms of depression [2, 5-10].

*Correspondence: mjafari@uci.edu

Department of Pharmaceutical Sciences, University of California Irvine, Irvine, CA 92697, USA
Although the traditional medicinal uses of $R$. rosea derive from Eastern Europe and Asia, $R$. rosea products have gained popularity worldwide among athletes as a natural remedy to prevent fatigue and improve performance [11]. We reported that $R$. rosea significantly extended both mean (24\%, both sexes) and maximum (16\% in males, $31 \%$ in females) lifespan of the fruit fly, Drosophila melanogaster $[12,13]$. The lifespan extension properties of $R$. rosea appear to be conserved among model species since the plant has been shown to extend the lifespan of worm and yeast models as well $[14,15]$. The mechanism of lifespan extension with $R$. rosea, however, remains to be determined. 
Drosophila melanogaster is emerging as an important model to examine the interactions between non-pathogenic microbes within the host. Since D. melanogaster can be easily manipulated genetically and experimentally, it can serve as a good model to enhance our understanding of animal-microbial symbiosis. Utilizing the Drosophila model system provides an integrative approach to study the relationship between an herbal extract supplementation and the impact it may have on the gut microbial composition. Another species of the Rhodiola family, Rhodiola crenulata, also exhibits multiple pharmacological traits like that of $R$. rosea, such as stress protection, neuroprotection, high altitude sickness mitigation, and anti-inflammatory activity [16-19]. Moreover, $R$. crenulata has been demonstrated to treat metabolic disorders in rats [20] and increase intracellular antimicrobial peptide expression while improving gut morphology in fruit flies [21]. Here we suspect that $R$. rosea may act like $R$. crenulata in that it may change the microbial composition of D. melanogaster. Additionally, R. rosea may mimic numerous other herbal therapies that have been reported to alleviate gastrointestinal and metabolic disorders, which are particularly prevalent in the process of agerelated microbial dysbiosis [22-24]. More specifically, the intestinal microbiota is significantly altered during severe age-related physiological ailments, such as obesity, insulin resistance, and general frailty, suggesting that agerelated changes in the gut may have an impact on overall healthspan and lifespan [25-27].

The average adult Drosophila intestine harbors only 5-20 microbial species which primarily belong to the families Enterobacteriaceae, Acetobacteraceae, and the order Lactobacillales [28-31]. Of these three strains, the only bacterial order present in considerable amounts in both Drosophila and mammals is Lactobacillales [3234]. When evaluating the microbial differences between fruit flies, it appears that the microbial content of $D$. melanogaster, independent of species uniformity, is similar between species that are fed on the same diet [35]. Conversely, more closely related species that feed on different diets are known to have a contrasting and diverse microbial compositions [35]. These findings suggest that certain bacterial families, such as Acetobacteraceae, may favor the low $\mathrm{pH}$ and high ethanol conditions present in fermenting fruits, thus influencing the microbiota of flies which favor fruit based diets [35].

The purpose of this work was to examine whether the anti-aging properties of $R$. rosea are due to its impact on the microbial composition of the fly gut. To date, there have been no published studies highlighting the impact of anti-aging botanical extracts on the microbial composition of the gut. These results will aim to build support for investigating the effects of botanical extracts on the gut microbiota and how they may help prevent against age-related intestinal diseases.

\section{Methods \\ Fly strain and treatment}

Oregon-R flies were obtained from the Bloomington Drosophila Stock Center at Indiana University. Rhodiola rosea extract (SHR-5) was obtained from the Swedish Herbal Institute. An independent HPLC analysis of this extract was performed by Alkemists Pharmaceuticals (Costa Mesa, CA) and this formulation was found to contain $1.7 \%$ salidroside and $4.5 \%$ total rosavins (data on file).

\section{Feeding}

Food composition and detailed housing techniques are described in Schriner et al. and Jafari et al. [13, 36].

Flies used in these assays were raised from larvae (5080 eggs per vial) in $5 \mathrm{~mL}$ of standard banana-molasses food composed of a $9 \%$ carbohydrate and a 3.6\% yeast content. Upon hatching from pupae, flies were transferred to autoclaved jars at a density of 300 per jar (150 males and 150 females) and separated by treatment. Rhodiola rosea $(25 \mathrm{mg} / \mathrm{mL})$ was supplied to the adults by mixing with the yeast solution (4\% yeast and $1 \%$ acetic acid) and was overlaid on top of the banana-molasses food while control flies only received the yeast solution with the food. $400 \mu \mathrm{L}$ of both the yeast solution containing treatment as well as the non-treatment yeast solution was added on the food. Survivors were counted every 2 days and transferred to newly autoclaved jars. The dose of $25 \mathrm{mg} / \mathrm{mL}$ was used as the optimal concentration as this dose has consistently resulted in lifespan extension in both sexes [12]. Flies were maintained at $22 \pm 1{ }^{\circ} \mathrm{C}$ under a $12 \mathrm{~h}$ light: $12 \mathrm{~h}$ dark cycle for all experiments.

\section{DNA extraction and qRT-PCR analysis}

Flies were separated into six groups of five $(n=5)$, per treatment and sex, and surface sterilized in $75 \%$ ethanol, $10 \%$ bleach, and DPBS, all for 1 min each. Flies were then homogenized and placed in $500 \mu \mathrm{L}$ of DPBS. DNA extractions were performed using the DNeasy Blood and Tissue Kit (Qiagen, West Sussex, UK) per manufacturer's protocol but with the addition of $20 \mathrm{mg} / \mathrm{mL}$ lysozyme. The extracted DNA was stored at $-4{ }^{\circ} \mathrm{C}$ before qRTPCR analysis. For estimation of Lactobacillus plantarum, Acetobacter pomorum, and 16S rDNA gene abundance, amplifications of each sample of extracted DNA was performed with each respective primer as described by Wong et al. [37]. The reaction mix comprised of $10 \mu \mathrm{L}$ Power SYBR green PCR master mix (Applied Biosystems), $2 \mu \mathrm{L} 10 \mu \mathrm{M}$ primers ( $1 \mu \mathrm{L}$ of each forward and reverse), $6 \mu \mathrm{L}$ of sterile water, and $2 \mu \mathrm{L}$ of approximately 25 ng DNA template in a $20 \mu \mathrm{L}$ volume, with reagents 
being used as the negative controls. Amplifications were conducted in a Miniopticon (Bio-Rad) with the following thermal profile: $95{ }^{\circ} \mathrm{C}$ for $5 \mathrm{~min}, 40$ amplification cycles of $95{ }^{\circ} \mathrm{C}$ for $15 \mathrm{~s}, 55.2^{\circ} \mathrm{C}$ for $30 \mathrm{~s}$, and $60^{\circ} \mathrm{C}$ for $30 \mathrm{~s}$, and a dissociation cycle of $95^{\circ} \mathrm{C}$ for $15 \mathrm{~s}, 60^{\circ} \mathrm{C}$ for $15 \mathrm{~s}$, and then brought back to $95{ }^{\circ} \mathrm{C}$. The average threshold cycle (Ct) values of two technical replicates per sample and primer set were calculated against a normalizing gene and quantification levels were calculated thereafter.

\section{Colony forming unit (CFU) analysis}

Bacterial growth plates were generated according to the following recipes:

Lactobacili MRS agar: $70 \mathrm{~g} / \mathrm{L}$ of BD Difco Lactobacili MRS agar

Nutrient agar: $5 \mathrm{~g} / \mathrm{L}$ peptone, $3 \mathrm{~g} / \mathrm{L}$ yeast, $15 \mathrm{~g} / \mathrm{L}$ agar, $5 \mathrm{~g} / \mathrm{L} \mathrm{NaCl}$.

All media were autoclaved at $121{ }^{\circ} \mathrm{C}$ for $15 \mathrm{~min}$. Flies were separated into six groups of five $(n=5)$, per treatment and sex, and surface sterilized sequentially in $10 \%$ bleach solution for $1 \mathrm{~min}, 75 \%$ ethanol for $1 \mathrm{~min}$, and PBS for $1 \mathrm{~min}$. Flies were then homogenized in $500 \mu \mathrm{L}$ of PBS. A series of dilutions were performed in order to have quantifiable number of colonies, which vary depending on the age of the fly. $50 \mu \mathrm{L}$ of diluted fly homogenate was plated on each media and spread evenly. The plates were then incubated at $28^{\circ} \mathrm{C}$ for $48-76 \mathrm{~h}$. Plates were scanned with an Epson v600 scanner and analyzed with ImageJ [38].

\section{Antimicrobial assays}

BD BBl Prepared Plated Media (Mueller-Hinton II Agar) was purchased from Fisher Scientific. Culturable bacteria isolated from both the environment, control, and treated flies were picked individually and grown overnight at $37{ }^{\circ} \mathrm{C}$ in Luria-Bertani broth media and then diluted until an OD600 measurement of 0.08-0.1 was observed, corresponding to a $0.5 \mathrm{McF}$ arland standard and $1.5 \times 10^{8}$ $\mathrm{CFU} / \mathrm{mL}$. The incubation temperature of $37{ }^{\circ} \mathrm{C}$ sufficiently promotes the growth of Microbacterium, Bacillus, and Lactococcus. The resulting media was plated on Mueller-Hinton II agar and then treated with $20 \mu \mathrm{L}$ of $R$. rosea, Kanamycin (positive control), and DI water on disks of autoclaved Whatman filter paper \#1. Concentrations of $R$. rosea used were 100, 50, 15, 10 and $1.5 \mathrm{mg} /$ $\mathrm{mL}$. The concentration of Kanamycin used was $1.5 \mathrm{mg} /$ $\mathrm{mL}$. Plates were incubated at $37{ }^{\circ} \mathrm{C}$ for another $24 \mathrm{~h}$ and then scanned with an Epson v600 scanner and analyzed with ImageJ [38]. The bacteria originally used for this assay was identified by $16 \mathrm{~S}$ rRNA Sanger sequencing (GENEWIZ) and the resulting raw data chromatograms were visualized by using the Chromas Pro software (Technolysium Ltd.) and then identified with BLASTN $(2.7 .1+)$ searches [39].

\section{S rRNA gene sequencing}

All samples were DNA extracted, amplified, and sequenced by the Integrated Microbiome Resource lab (IMR) at Dalhousie University (Halifax, Canada). In brief, DNA was extracted from $5 \mathrm{mg}$ of frozen flies per sample using the QIAamp PowerFecal DNA Kit (Qiagen) per manufacturer's protocol. All DNA samples were amplified by PCR targeting the 16S rRNA gene sequence (regions V6-V8) as previously demonstrated [40], and libraries were prepared by following the guidelines provided by Illumina (San Diego, USA; Part \#15044223, Rev. B). The amplified 16S rDNA fragments were then sequenced using the Illumina MiSeq platform by using the Microbiome Helper workflow [40]. Raw sequences were analyzed with QIIME (Quantitative Insight Into Microbial Ecology) and FastQC (v0.11.5) coupled with PEAR (v0.9.10) was used to evaluate raw reads, identify ambiguous reads, and stitch the reads together [41-43]. Chimeric DNA molecules were screened using VSEARCH (v1.11.1) and removed with the UCHIME algorithm [44, 45]. Open-reference OTU (Operational taxonomic units) picking was performed at $97 \%$ identity using SortMeRNA and SUMACLUST and reads were clustered against the Greengenes database [46-48]. Low confidence OTUs were removed with a $0.1 \%$ threshold and the final OTU table was normalized per sample using DESeq2 [49].

\section{Statistical analysis}

Parametric unpaired $t$ tests with Welch's correction were used to display statistical and graphical representations of the qRT-PCR and CFU data using GraphPad Prism version 7.00 for Mac OS X, GraphPad Software, La Jolla California USA, http://www.graphpad.com. Box-andwhisker plots were created to display the 25th to 75th percentiles of the data sets with a line in the middle of the plot as the median. Minimum to maximum values are shown by the whiskers. The $16 \mathrm{~S}$ rRNA amplicon sequencing data was analyzed by converting the QIIME derived BIOM OTU table to a format compatible with STAMP (v2.1.3) [50]. Box plots were generated through STAMP to show the median of the data as a line, the mean of the data as a star, the 25th and 75th percentiles of the data as the top and bottom of the box, and whiskers to indicate the minimum and maximum values within $1.5^{*}$ (75th25th percentile) of the median. Data points outside of the whiskers are shown as crosses. The statistical hypothesis test used for these samples was a Welch's $t$ test with the Storey's FDR multiple test correction at 0.05 to control 
the false discovery rate [51]. Heatmap plots were generated through STAMP alongside PCA plots to show the proportion of sequences assigned to each feature with the use of a dendrogram to cluster features and samples.

\section{Results \\ Rhodiola rosea significantly alters the microbial composition}

The objective of this work was to study whether $R$. rosea changes the microbial composition of the fruit fly throughout its lifespan. After hatching from their pupae (Day 0), we housed the control flies and the $R$. rosea fed flies separately and started the experiment. The flies were placed on new media every other day and assayed at days 10 and 40, corresponding to 'young' and 'old' in respect to the fly strain used. The relative bacterial abundances generated from the flies were identified by $16 \mathrm{~S}$ rRNA amplicon sequencing. It has been previously reported that the bacterial species which dominate the gut of young flies belong to the genus Lactobacillus, while the bacteria that dominate the gut of older flies belong to the genus Acetobacter [52]. However, our study revealed that classes Bacilli and Alphaproteobacteria, which include the genera Lactobacillus and Acetobacter, respectively, display different abundances of each class of bacteria at younger ages when compared to a prior study [52]. We observed an increased ratio of Alphaproteobacteria (67.23\%) to Bacilli (31.78\%) in 10 days old flies (Fig. 1a). In 40 days old flies, we observed a similar ratio, with Alphaproteobacteria $(80.91 \%)$ dominating in abundance, displaying a trend that is similar to previous studies (Fig. 2a) [52].

When comparing $R$. rosea fed flies to control flies, the relative abundances of several individual bacterial taxa was differentially associated between each respective age group given treatment (Figs. $1 \mathrm{~b}$ and $2 \mathrm{~b}$ ). Most of the taxa in the flies derived from the phyla Firmicutes and Proteobacteria (Figs. 1b and 2b). The presence of Gammaproteobacteria, which is an indication of dysbiosis in the fly gut [53], was present at no levels in the guts of 10 days old flies (Fig. 1b), but was present at minimal levels in only 2 samples (Control1 and Control4) in 40 days old flies (Fig. 2b). The genus Lactococcus (individual graph not shown) was more abundant in the guts of control flies for both time points (Figs. $1 \mathrm{~b}$ and $2 \mathrm{~b}$ ), but only 40 days old flies given $R$. rosea treatment displayed significantly lower presence of this genus $(p=0.019$, Welch's $t$ test with Storey FDR multiple test correction). The genus Enterococcus was minimally present in the guts of 40 days old flies (Fig. 2b), but showed no statistical significance when compared between control and treatment groups ( $p>0.05$, Welch's t-test with Storey FDR multiple test correction).

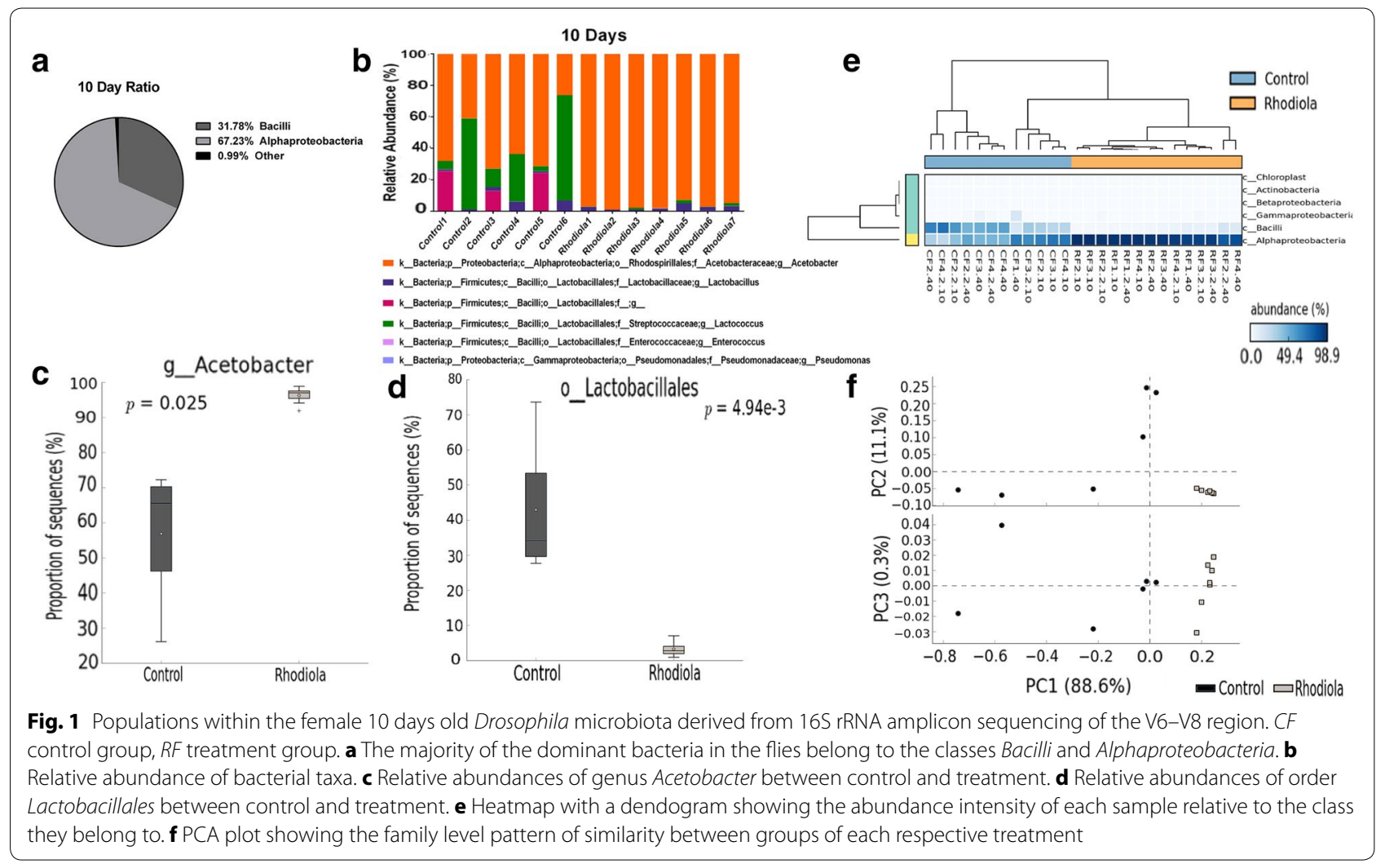




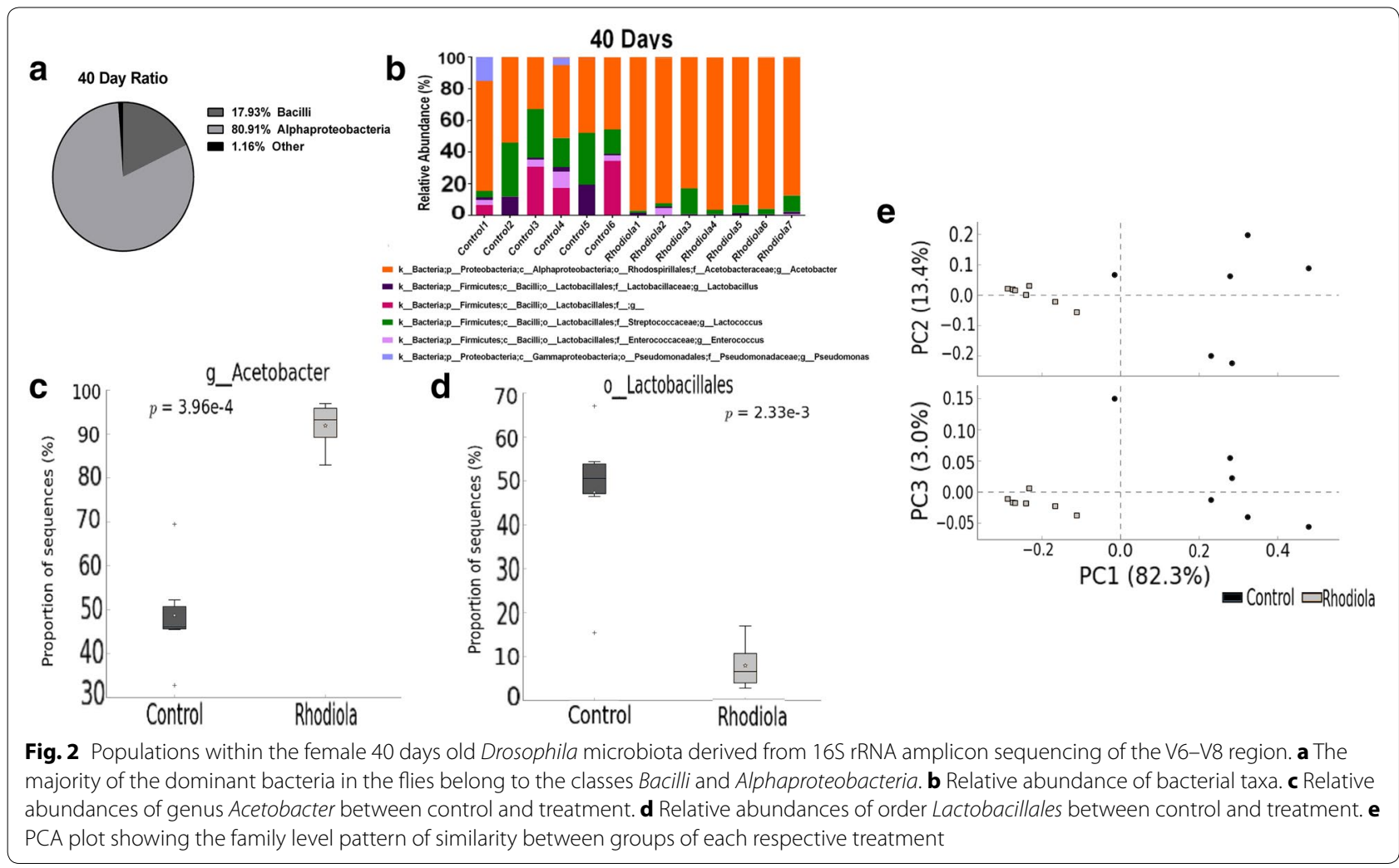

The genus Acetobacter and the order Lactobacillales showed stark differences between control and treatment groups at both ages. At both 10 and 40 days, the levels of Acetobacter were significantly increased with $R$. rosea treatment, while levels of Lactobacillales were significantly decreased (Figs. 1c, d and 2c, d). A generated heatmap plot shows the intensity of abundance between control and $R$. rosea fed flies across various classes of bacteria (Fig. 1e). All control samples (indicated by a ' $C$ ' in front of the sample name) displayed abundance intensities for both Bacilli and Alphaproteobacteria classes, while all $R$. rosea samples (indicated by a 'R' in front of the sample name) displayed stronger abundance intensities for the class Alphaproteobacteria but lower intensities for the class Bacilli. Principal components analysis (PCA) plots were generated for both age groups by using the Euclidean distance as the dissimilarity metric to display the spatial variation of control and treatment groups across three principle axes (PC1, PC2, and PC3) (Figs. 1f and 2e).

\section{Rhodiola rosea alters the relative amounts of individual species abundance}

In order to analyze the bacterial genera in more detail, quantitative real-time PCR (qRT-PCR) was utilized to evaluate the species level differences in our samples. Total bacterial content (measured through the $16 \mathrm{~S}$ rDNA gene) as well as the relative abundances of two highly relevant bacterial species, L. plantarum and A. pomorum, were significantly altered with treatment of $R$. rosea (Fig. 3ac). Total bacterial load was increased with treatment of R. rosea in females at both 10 and 40 days of age (Fig. 3a). $16 \mathrm{~S}$ rDNA of the V3 hypervariable region showed significant differences between control and treatment with $\mathrm{p}$ values of 0.0026 and 0.0088 in 10 and 40 days, respectively (Fig. 3a). Additionally, A. pomorum was present at lower levels in $R$. rosea fed flies at 10 days of age, but present at higher levels with $R$. rosea supplementation at 40 days of age (Fig. 3b). A. pomorum differences between control and treatment resulted in p values of 0.0174 and 0.0178 in 10 and 40 days, respectively (Fig. 3b). Bacterial species belonging to $L$. plantarum were present at lower levels at both stages of the fly lifespan given treatment (Fig. 3c). L. plantarum differences between control and treatment resulted in $\mathrm{p}$ values of 0.0001 and 0.0074 in 10 and 40 days, respectively (Fig. 3c). Statistics were performed using the unpaired $t$ test with Welch's correction. Each group contained samples with $n=5$ with four technical replicates each within 2 biological replicates. 

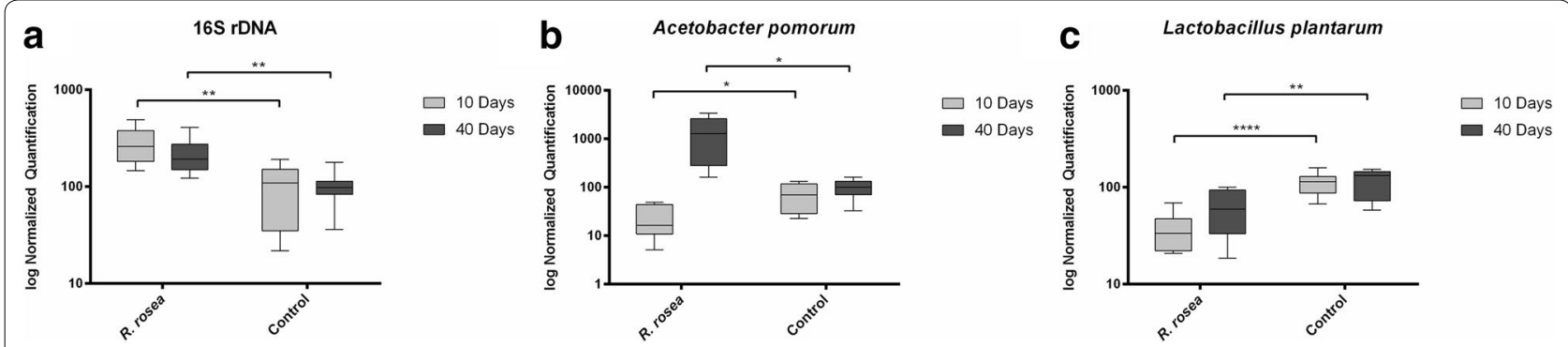

Fig. 3 Real-time quantitative PCR results for 10 and 40 days old female flies. ${ }^{*} p<0.05$; ${ }^{* *} p<0.01$. a $16 \mathrm{~S}$ rDNA of the V3 hypervariable region and the species $\mathbf{b}$ A. pomorum and $\mathbf{c}$ L. plantarum showed statistically significant differences

\section{Rhodiola rosea decreases culturable bacterial load}

To assess the amount of culturable bacterial load, homogenized flies were plated on both De Man, Rogosa and Sharpe (MRS) and nutrient agar. MRS agar has been established as the conventional bacterial media to cultivate microorganisms belonging to the Lactobacillus genus [54]. At earlier ages, the fly gut exhibits minimal colonization with microorganisms, thus resulting in a decreased bacterial load [30]. We showed here that the earlier stages of the fly life consisted of a lower amount of culturable bacteria, corresponding to less colony forming units (CFUs) (Fig. 4a). More importantly, $R$. rosea treatment significantly reduced the amount of CFUs in 10 days old flies plated on MRS media (Fig. 4a). Additionally, we observed that $R$. rosea decreased the amount of CFUs in 40 days old flies plated on both MRS and nutrient agar. The difference in CFUs between $R$. rosea and control fed flies at 40 days was more prominent in both media (30,000 CFUs) due to the exponential growth of bacteria that inhabit the later stages of the fly gut (Fig. 4a). Statistics were performed using the unpaired $t$ test with Welch's correction. For CFU testing, each group contained samples with $\mathrm{n}=5$ with six technical replicates each within one biological replicate.

\section{Rhodiola rosea does not have antimicrobial properties against Microbacterium, Bacillus, and Lactococcus}

Multiple studies have demonstrated that plant extracts that exhibit zones of inhibition with diameters $10 \mathrm{~mm}$ may possess antimicrobial properties [55, 56]. Rhodiola rosea has previously been shown to exhibit antimicrobial activity on multiple strains of Staphylococcus aureus, but the source of growth (botanical garden in Poznan, Poland) and the composition of the extract (2.04\% salidroside and $1.46 \%$ cinnamyl alcohol) were different than the $R$. rosea that was used in our study [57]. We performed Kirby-Bauer disk diffusion tests with $R$. rosea concentrations of $1.5,15,50$, and $100 \mathrm{mg} / \mathrm{mL}$ with a positive control of Kanamycin $(1500 \mu \mathrm{g} / \mathrm{mL})$ and a negative control of DI water. The amount of total $R$. rosea extract added to each disk was 30, 300, 1000, and $2000 \mu \mathrm{g}$ which corresponds to the concentrations above, with a total amount of $30 \mu \mathrm{g}$ Kanamycin. We plated bacterial isolates grown in our lab belonging to the genera Microbacterium (Fig. 4b), Bacillus (Fig. 4c), and Lactococcus (Fig. 4d) against the previously mentioned concentrations of $R$. rosea. All concentrations of $R$. rosea appeared to not display zones of inhibition when plated across all three bacterial genera (Fig. $4 \mathrm{~b}-\mathrm{d}$ ).

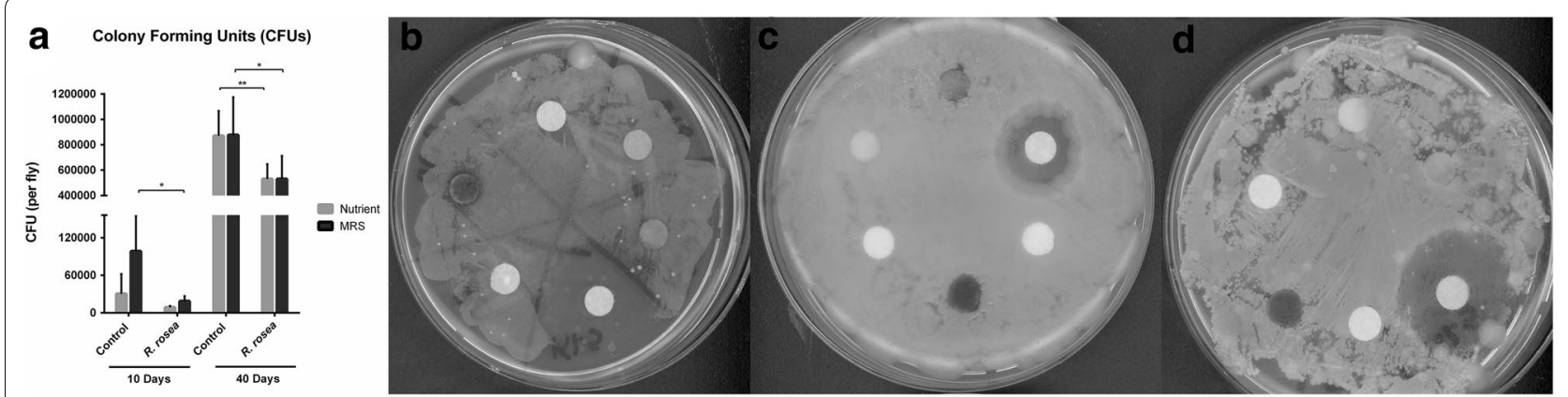

Fig. 4 a Colony forming units of 10 and 40 days old female flies when plated on MRS and nutrient agar. ${ }^{*} p<0.05$; **p $<0.01$. Kirby-Bauer antimicrobial assays used to test genera $\mathbf{b}$ Microbacterium, $\mathbf{c}$ Bacillus, and $\mathbf{d}$ Lactococcus against $R$. rosea infused disks 


\section{Discussion}

The aim of this study was to determine whether $R$. rosea can change the gut microbial community of $D$. melanogaster. Our group had previously reported that the root extract of $R$. rosea extends the lifespan and improves the healthspan of the fruit fly, but the exact underlying mechanisms of lifespan extension remains unclear [12, $13,36]$. In this study, we examined the impact of $R$. rosea on the microbial dynamics of the fly gut and whether changing the gut microbiome could be beneficial for host longevity. When evaluating the impact of $R$. rosea on the fly gut microbiota, we observed sex specific differences between fly groups which could be contributed to a variety of physiological factors. At adult stages, female fruit flies require a greater protein intake needed for egg production, thus consuming more environmental yeast when compared to their male counterparts [58]. Due to extensive contact with environmental nutrients, the female flies, along with their microbial communities, experience metabolism-related shifts through alteration of host signaling pathways [59]. Performing 16S rRNA sequencing exclusively on female fruit flies allowed for investigation into the environmental and nutrient microbe-altering effects of $R$. rosea and how it influenced the microbial community of the host. Studies involving both sexes and multiple strains of Drosophila will be required to thoroughly understand the paired effect of $R$. rosea and yeast consumption on the host microbiota.

Our results show that while control female Oregon-R fruit flies establish and maintain a consistent microbial composition throughout their lifespan, the $R$. rosea supplemented flies maintained a microbial composition which differed in relative abundance of order Lactobacillales and genus Acetobacter when compared to control (Figs. $1 \mathrm{~b}$ and $2 \mathrm{~b}$ ). These changes, with respect to supplementation of $R$. rosea, are likely to vary between Drosophila strains, with additional factors influencing the microbiota such as the nutritional composition and sex [35, 60-62]. Male flies in our study displayed no significant changes in L. plantarum, A. pomorum, and the $16 \mathrm{~S}$ rDNA gene when supplemented with $R$. rosea $(\mathrm{p}>0.05$, Unpaired Welch's t test) (Additional file 1: Figure S1a-c). $\mathrm{CFU}$ tests revealed that male flies displayed significant decrease in CFU counts at early ages of their lifespan (p value $=0.0096$ for MRS, $p$ value $=0.0367$ for nutrient), but no difference was observed at the later stages of their lifespan, where flies experience an increased bacterial load (Additional file 1: Figure S1d).

Our 16S rRNA amplicon sequencing identified the differences in diversity between control and $R$. rosea fed flies. In 10 days old flies, control flies had an average Operational Taxonomic Unit (OTU) count of 18.17, while $R$. rosea fed flies had a count of 16.14 (Additional file 2: Table S1). In 40 days old flies, control flies had a OTU count of 22.33, while $R$. rosea fed flies had a count of 17.7 (Additional file 2: Table S1). Although we observe a decrease in bacterial diversity in $R$. rosea fed flies, the total abundance of bacteria increase, as indicated through $16 \mathrm{~S}$ rDNA qRT-PCR analysis (Fig. 3a). Although our results suggest that the $R$. rosea induced gut microbiome changes are age-dependent, to fully comprehend the time point where $R$. rosea begins to induce such changes, additional time points (i.e. time of eclosion) need to be evaluated. Furthermore, since several samples in our study missed certain bacterial genera (Lactococcus, Enterococcus), additional samples at various time points need to be evaluated to determine which microorganisms are natively present in the gut in comparison to which are acquired from the environment. To limit the impact on external inputs from contributing to the bacterial load within the Drosophila gut, many studies have utilized germ-free flies as a model to test the effect of individual bacteria on host physiology [63, 64]. Utilizing the gnotobiotic model will allow us to control the influence of environmental factors to discern how $R$. rosea directly affects individual bacterial species inside the host.

The most notable observations in this study resulted from the ability of $R$. rosea to increase the ratio of genus Acetobacter and decrease the order Lactobacillales at both the early and later stages of the fly lifespan (Figs. 1c, $\mathrm{d}$ and $2 \mathrm{c}, \mathrm{d})$. Observations were taken at the order level due to the presence of unidentified reads that belong to the families and genera under the Lactobacillales order. When comparing the $16 \mathrm{~S}$ rRNA sequencing data with the 16S rDNA qRT-PCR reads, we noticed that although the genus Acetobacter was increased in 10 days old flies that were fed $R$. rosea, the species $A$. pomorum was significantly decreased in these flies (Figs. $1 \mathrm{~b}$ and $3 \mathrm{~b}$ ). This contrast is possible due to the presence of other commensal species belonging to the genus Acetobacter, such as Acetobacter pasteurianus, Acetobacter aceti, and Acetobacter tropicalis [65]. We observed an opposite trend between treatment groups when comparing between the $16 \mathrm{~S}$ rDNA amplification and CFU counts. A decrease in CFUs corresponded with an increase in 16S rDNA expression, indicating that $R$. rosea fed flies experience a lower culturable bacterial load but more overall bacteria (Figs. 3a and 4a). Interestingly enough, both MRS and nutrient agar displayed parallel decreases in bacterial load when $R$. rosea fed flies were plated, demonstrating the similarities between the bacteria that are culturable when utilizing the non-selective nature of the nutrient media. A significant decrease in the CFUs with 10 days old flies fed $R$. rosea on MRS also suggests that Lactobacillus is responsible for variation in culturable bacteria at the earlier stages of the fly lifespan (Fig. 4a). 
Previous reports demonstrated the impact of an altered diet such as changes in the sugar versus protein composition in the fly media affects the Acetobacter to Lactobacillus ratio in flies $[52,66]$. Since a change in the diet impacts host physiology, the effects of the diet on the microbial community suggest that the health of the host is a major determinant for shaping the gut microbial population in flies [67]. Although studies have reported that the commensal bacterial load fluctuates throughout the Drosophila lifespan, we observed the dominance of Acetobacter throughout all stages of the fly life in both control and R. rosea fed flies [52]. Since Acetobacter species thrive under fully aerobic conditions and Lactobacillus species are incapable of thriving in a ubiquitously oxygenated environment, we propose the possibility that the gut oxygen tension experiences a shift towards aerobic conditions after supplementation of $R$. rosea, thus promoting the growth of Acetobacter [32,68]. This is particularly more likely in older flies who consume more oxygen and produce a more severe physiological response to conventional oxygen intake when compared to their younger counterparts [69]. In addition to $R$. rosea playing a role in changing gut oxygen tension, we suspect the extract may further modify immune system function in the Drosophila gut. An altered gut microbial composition, as a result of the supplementation of $R$. rosea, may contribute towards limiting age-related dysplastic changes by positively modulating the process of mis-differentiation in intestinal stem cells (ISCs) and their progeny, leading to the improvement in intestinal function and subsequently benefitting the health of the host. Because epithelial barrier dysfunction is strongly associated with fly aging and mortality, we believe $R$. rosea may attenuate this process at the later stages of the fly life [70]. In summary, evaluating the impact of anti-aging botanical extracts, such as $R$. rosea, on the gut microbiome using $D$. melanogaster as a model system may provide a platform to understand the interactions between the microbiome, lifespan, and healthspan.

\section{Conclusions}

This study demonstrates the effectiveness of using $D$. melanogaster as a model to study the effect of anti-aging botanical extracts on the gut microbial community. We observed changes in the relative abundance of order $\mathrm{Lac}$ tobacillales and genus Acetobacter in the female fly lifespan. We also saw an increase in total bacterial load, and a decrease in OTU and CFU counts with supplementation of $R$. rosea. Future studies are needed to evaluate a potential link between major gut immune genes to bacterial diversity and abundance in order to thoroughly understand whether certain botanical extracts increase lifespan and improve healthspan by altering the gut microbiome.

\section{Additional files}

Additional file 1: Figure S1. qRT-PCR and CFU analysis of male D. melanogaster at early and late stages of the fly lifespan.

Additional file 2: Table S1. OTU table provided by $16 \mathrm{~S}$ rRNA amplicon sequencing of female $D$. melanogaster at early and late stages of the fly lifespan.

\section{Abbreviations}

PCA: principal components analysis; qRT-PCR: quantitative real-time PCR; MRS: De Man, Rogosa and Sharpe; CFUs: colony forming units; OTU: operational taxonomic units; Ct: threshold cycle; ISCs: intestinal stem cells.

\section{Authors' contributions}

Conceived and designed the experiments: KEL, EAS, SES, MJ. Performed the experiments: KEL, DK. Analyzed the data: KEL, DK, EAS, SES, MJ. Wrote the manuscript: KEL, DK, MJ. All authors read and approved the final manuscript.

\section{Acknowledgements}

We thank Dr. Anthony Long $(\mathrm{UCl})$ for providing the fly strain. We also thank Dr. Claudia Weihe (UCI) and Dr. Katrine Whiteson (UCI) and Dr. Parvin Shahrestani (California State University, Fullerton) for their expert advice and insight on the methodology of this work.

Competing interests

The authors declare that they have no competing interests.

Availability of data and materials

The authors declare that all data supporting the findings of this study are available within the article.

Consent for publication

Not applicable.

Ethics approval and consent to participate

Not applicable.

\section{Funding}

This work was supported by an Undergraduate Research Opportunity Program grant from the University of California, Irvine.

\section{Publisher's Note}

Springer Nature remains neutral with regard to jurisdictional claims in published maps and institutional affiliations.

Received: 8 January 2018 Accepted: 12 March 2018

Published online: 20 March 2018

\section{References}

1. Mao JJ, Xie SX, Zee J, Soeller I, Li QS, Rockwell K, Amsterdam JD. Rhodiola rosea versus sertraline for major depressive disorder: a randomized placebo-controlled trial. Phytomedicine. 2015;22(3):394-9.

2. Olsson EM, von Scheele B, Panossian AG. A randomised, double-blind, placebo-controlled, parallel-group study of the standardised extract shr-5 of the roots of Rhodiola rosea in the treatment of subjects with stressrelated fatigue. Planta Med. 2009;75(2):105-12

3. Panossian A, Wikman G. Evidence based efficacy and effectiveness of Rhodiola SHR-5 extract in treating stress- and age-associated disorders. In: Cuerrier A, Kwesi AN, editors. Traditional herbal medicines for modern times. Boca Raton: CRC Press; 2014. p. 203-21.

4. Panossian A, Wikman G, Sarris J. Rosenroot (Rhodiola rosea): traditional use, chemical composition, pharmacology and clinical efficacy. Phytomedicine. 2010;17(7):481-93. 
5. Saratikov AS, Krasnov EA, Chnikina LA, Duvidson LM, Sotova MI, Marina TF, Nechoda MF, Axenova RA, Tscherdinzeff SG. Rhodiolosid, a new glycoside from Rhodiola rosea and its pharmacological properties. Pharmazie. 1968;23:392-5.

6. Darbinyan V, Kteyan A, Panossian A, Gabrielian E, Wikman G, Wagner H. Rhodiola rosea in stress induced fatigue-a double blind cross-over study of a standardized extract SHR-5 with a repeated low-dose regimen on the mental performance of healthy physicians during night duty. Phytomedicine. 2000;7(5):365-71.

7. Shevtsov VA, Zholus BI, Shervarly VI, Vol'skij VB, Korovin YP, Khristich MP, Roslyakova NA, Wikman G. A randomized trial of two different doses of a SHR-5 Rhodiola rosea extract versus placebo and control of capacity for mental work. Phytomedicine. 2003;10(2-3):95-105.

8. De Bock K, Eijnde BO, Ramaekers M, Hespel P. Acute Rhodiola rosea intake can improve endurance exercise performance. Int J Sport Nutr Exerc Metab. 2004;14(3):298-307.

9. Spasov AA, Wikman GK, Mandrikov VB, Mironova IA, Neumoin W. A double-blind, placebo-controlled pilot study of the stimulating and adaptogenic effect of Rhodiola rosea SHR-5 extract on the fatigue of students caused by stress during an examination period with a repeated low-dose regimen. Phytomedicine. 2000;7(2):85-9.

10. Darbinyan V, Aslanyan G, Amroyan E, Gabrielyan E, Malmstrom C, Panossian A. Clinical trial of Rhodiola rosea L. extract SHR-5 in the treatment of mild to moderate depression. Nord J Psychiatry. 2007;61(5):343-8.

11. Parisi A, Tranchita E, Duranti G, Ciminelli E, Quaranta F, Ceci R, Cerulli C, Borrione P, Sabatini S. Effects of chronic Rhodiola Rosea supplementation on sport performance and antioxidant capacity in trained male: preliminary results. J Sports Med Phys Fitness. 2010;50(1):57-63.

12. Schriner SE, Abrahamyan A, Avanessian A, Bussel I, Maler S, Gazarian M, Holmbeck MA, Jafari M. Decreased mitochondrial superoxide levels and enhanced protection against paraquat in Drosophila melanogaster supplemented with Rhodiola rosea. Free Radic Res. 2009;43(9):836-43.

13. Jafari M, Felgner JS, Bussel II, Hutchili T, Khodayari B, Rose MR, Vince-Cruz C, Mueller LD. Rhodiola: a promising anti-aging Chinese herb. Rejuvenation Res. 2007;10(4):587-602.

14. Wiegant FA, Surinova S, Ytsma E, Langelaar-Makkinje M, Wikman G, Post JA. Plant adaptogens increase lifespan and stress resistance in C. elegans. Biogerontology. 2009;10(1):27-42.

15. Bayliak MM, Lushchak VI. The golden root, Rhodiola rosea, prolongs lifespan but decreases oxidative stress resistance in yeast Saccharomyces cerevisiae. Phytomedicine. 2011;18(14):1262-8.

16. Lee $\mathrm{OH}$, Kwon Yl, Apostolidis E, Shetty K, Kim YC. Rhodiola-induced inhibition of adipogenesis involves antioxidant enzyme response associated with pentose phosphate pathway. Phytother Res. 2011;25(1):106-15.

17. Qu ZQ, Zhou Y, Zeng YS, Lin YK, LiY, Zhong ZQ, Chan WY. Protective effects of a Rhodiola crenulata extract and salidroside on hippocampal neurogenesis against streptozotocin-induced neural injury in the rat. PLoS ONE. 2012;7(1):e29641

18. Lee SY, Li MH, Shi LS, Chu H, Ho CW, Chang TC. Rhodiola crenulata extract alleviates hypoxic pulmonary edema in rats. Evid Based Complement Alternat Med. 2013;2013:718739.

19. Pooja, Bawa AS, Khanum F. Anti-inflammatory activity of Rhodiola rosea"a second-generation adaptogen". Phytother Res. 2009;23(8):1099-102.

20. Wang J, Rong X, Li W, Yang Y, Yamahara J, Li Y. Rhodiola crenulata root ameliorates derangements of glucose and lipid metabolism in a rat model of the metabolic syndrome and type 2 diabetes. J Ethnopharmacol. 2012;142(3):782-8.

21. Zhu C, Guan F, Wang C, Jin LH. The protective effects of Rhodiola crenulata extracts on Drosophila melanogaster gut immunity induced by bacteria and SDS toxicity. Phytother Res. 2014;28(12):1861-6.

22. Comar KM, Kirby DF. Herbal remedies in gastroenterology. J Clin Gastroenterol. 2005;39(6):457-68.

23. Langmead L, Rampton DS. Review article: complementary and alternative therapies for inflammatory bowel disease. Aliment Pharmacol Ther. 2006;23(3):341-9.

24. Han GC, Ko SK, Sung JH, Chung SH. Compound Kenhances insulin secretion with beneficial metabolic effects in $\mathrm{db} / \mathrm{db}$ mice. J Agric Food Chem. 2007;55(26):10641-8.

25. Kallus SJ, Brandt LJ. The intestinal microbiota and obesity. J Clin Gastroenterol. 2012;46(1):16-24.
26. De Bandt JP, Waligora-Dupriet AJ, Butel MJ. Intestinal microbiota in inflammation and insulin resistance: relevance to humans. Curr Opin Clin Nutr Metab Care. 2011;14(4):334-40.

27. Claesson MJ, Jeffery IB, Conde S, Power SE, O'Connor EM, Cusack S, Harris HM, Coakley M, Lakshminarayanan B, O'Sullivan O, Fitzgerald GF, Deane J, O'Connor M, Harnedy N, O'Connor K, O'Mahony D, van Sinderen D, Wallace M, Brennan L, Stanton C, Marchesi JR, Fitzgerald AP, Shanahan F, Hill C, Ross RP, O'Toole PW. Gut microbiota composition correlates with diet and health in the elderly. Nature. 2012;488(7410):178-84.

28. Ryu JH, Kim SH, Lee HY, Bai JY, Nam YD, Bae JW, Lee DG, Shin SC, Ha EM, Lee WJ. Innate immune homeostasis by the homeobox gene caudal and commensal-gut mutualism in Drosophila. Science. 2008;319(5864):777-82.

29. Brummel T, Ching A, Seroude L, Simon AF, Benzer S. Drosophila lifespan enhancement by exogenous bacteria. Proc Natl Acad Sci USA. 2004;101(35):12974-9.

30. Ren C, Webster P, Finkel SE, Tower J. Increased internal and external bacterial load during Drosophila aging without life-span trade-off. Cell Metab. 2007;6(2):144-52.

31. Cox CR, Gilmore MS. Native microbial colonization of Drosophila melanogaster and its use as a model of Enterococcus faecalis pathogenesis. Infect Immun. 2007;75(4):1565-76.

32. Ljungh A, Wadstrom T. Lactobacillus molecular biology: from genomics to probiotics. Norfolk: Caister Academic Press: 2009

33. Hammes WP, Hertel C. The genera Lactobacillus and Carnobacterium. In: Falkow S, Rosenberg E, Schleifer KH, Stackebrandt E, Dworkin M, editors, The Prokaryotes: Archaea. Bacteria: Firmicutes, Actinomycetes. New York: Springer Science + Business Media, LLC; 2007. p. 320-403.

34. Gilmore MS. The Enterococci: pathogenesis, molecular biology, and antibiotic resistance. Washington, DC: ASM Press; 2002.

35. Chandler JA, Lang JM, Bhatnagar S, Eisen JA, Kopp A. Bacterial communities of diverse Drosophila species: ecological context of a host-microbe model system. PLoS Genet. 2011;7(9):e1002272.

36. Schriner SE, Lee K, Truong S, Salvadora KT, Maler S, Nam A, Lee T, Jafari M. Extension of Drosophila lifespan by Rhodiola rosea through a mechanism independent from dietary restriction. PLoS ONE. 2013;8(5):e63886.

37. Wong $A C$, Luo $Y$, Jing $X$, Franzenburg $S$, Bost A, Douglas AE. The Host as the driver of the microbiota in the gut and external environment of Drosophila melanogaster. Appl Environ Microbiol. 2015;81(18):6232-40.

38. Schneider CA, Rasband WS, Eliceiri KW. NIH Image to ImageJ: 25 years of image analysis. Nat Methods. 2012;9(7):671-5.

39. Altschul SF, Madden TL, Schaffer AA, Zhang J, Zhang Z, Miller W, Lipman DJ. Gapped BLAST and PSI-BLAST: a new generation of protein database search programs. Nucleic Acids Res. 1997:25(17):3389-402.

40. Comeau AM, Douglas GM, Langille MG. Microbiome helper: a custom and streamlined workflow for microbiome research. mSystems. 2017;2(1):e00127-16.

41. Caporaso JG, Kuczynski J, Stombaugh J, Bittinger K, Bushman FD, Costello EK, Fierer N, Pena AG, Goodrich JK, Gordon JI, Huttley GA, Kelley ST, Knights D, Koenig JE, Ley RE, Lozupone CA, McDonald D, Muegge BD, Pirrung M, Reeder J, Sevinsky JR, Turnbaugh PJ, Walters WA, Widmann J, Yatsunenko T, Zaneveld J, Knight R. QIIME allows analysis of high-throughput community sequencing data. Nat Methods. 2010;7(5):335-6.

42. Andrews, S. FastQC: a quality control tool for high throughput sequence data; 2010. http://www.bioinformatics.babraham.ac.uk/projects/fastqc. Accessed 17 Nov 2017.

43. Zhang J, Kobert K, Flouri T, Stamatakis A. PEAR: a fast and accurate Illumina Paired-End reAd mergeR. Bioinformatics. 2014;30(5):614-20.

44. Rognes T, Flouri T, Nichols B, Quince C, Mahe F. VSEARCH: a versatile open source tool for metagenomics. PeerJ. 2016:4:e2584.

45. Edgar RC, Haas BJ, Clemente JC, Quince C, Knight R. UCHIME improves sensitivity and speed of chimera detection. Bioinformatics. 2011;27(16):2194-200.

46. Kopylova E, Noe L, Touzet H. SortMeRNA: fast and accurate filtering of ribosomal RNAs in metatranscriptomic data. Bioinformatics. 2012:28(24):3211-7.

47. Mercier C, Boyer F, Bonin A, Coissac E. SUMATRA and SUMACLUST: fast and exact comparison and clustering of sequences. In: Programs and Abstracts of the SeqBio 2013 workshop (Abstract), GdRBIM and gdrIM Montpellier, France. 2013; 27-29. http://metabarcoding.org/sumatra. Accessed 17 Nov 2017. 
48. DeSantis TZ, Hugenholtz P, Larsen N, Rojas M, Brodie EL, Keller K, Huber T, Dalevi D, Hu P, Andersen GL. Greengenes, a chimera-checked $16 \mathrm{~S}$ rRNA gene database and workbench compatible with ARB. Appl Environ Microbiol. 2006;72(7):5069-72.

49. Love MI, Huber W, Anders S. Moderated estimation of fold change and dispersion for RNA-seq data with DESeq2. Genome Biol. 2014;15(12):550.

50. Parks DH, Tyson GW, Hugenholtz P, Beiko RG. STAMP: statistical analysis of taxonomic and functional profiles. Bioinformatics. 2014;30(21):3123-4.

51. Storey JD, Taylor JE, Siegmund D. Strong control, conservative point estimation and simultaneous conservative consistency of false discovery rates: a unified approach. J R Stat Soc Ser B (Stat Method). 2004;66:187-205.

52. Wong CN, Ng P, Douglas AE. Low-diversity bacterial community in the gut of the fruitfly Drosophila melanogaster. Environ Microbiol. 2011;13(7):1889-900.

53. Clark RI, Salazar A, Yamada R, Fitz-Gibbon S, Morselli M, Alcaraz J, Rana A, Rera M, Pellegrini M, Ja WW, Walker DW. Distinct shifts in microbiota composition during Drosophila aging impair intestinal function and drive mortality. Cell Rep. 2015;12(10):1656-67.

54. De Man JC, Rogosa M, Sharpe ME. A medium for the cultivation of Lactobacili. J Appl Bacteriol. 1960;23:130-5.

55. Zwadyk P. Enteriobacteriaceae in zinsser microbiology. 20th ed. Stuttgart: George ThieneVerlag; 1972. p. 20-32.

56. Usman $\mathrm{H}$, Osuji JC. Phytochemical and in vitro antimicrobial assay of the leaf extract of Newbouldia laevis. Afr J Tradit Complement Altern Med. 2007;4(4):476-80.

57. Furmanowa M, Starościak B, Lutomski J, Kozłowski J, Urbańska N, Krajewska-Patan A, Pietrosiuk A, Szypuła W. Antimicrobial effect of Rhodiola rosea $\mathrm{L}$. roots and callus extracts on some strains of Staphylococcus aureus. Herba Pol. 2002;48:23.

58. Newland PL, Cobb M, Marion-Poll F. Insect taste. London: Taylor \& Francis; 2008

59. Wong AC, Dobson AJ, Douglas AE. Gut microbiota dictates response of Drosophila to diet. J Exp Biol. 2014;217(Pt 11):1894-901.
60. Staubach F, Baines JF, Kunzel S, Bik EM, Petrov DA. Host species and environmental effects on bacterial communities associated with Drosophila in the laboratory and in the natural environment. PLoS ONE. 2013;8(8):e70749.

61. Wong AC, Chaston JM, Douglas AE. The inconstant gut microbiota of Drosophila species revealed by 165 rRNA gene analysis. ISME J. 2013;7(10):1922-32.

62. Han G, Lee HJ, Jeong SE, Jeon CO, Hyun S. Comparative analysis of Dros ophila melanogaster gut microbiota with respect to host strain, sex, and age. Microb Ecol. 2017;74(1):207-16.

63. Shin SC, Kim SH, You H, Kim B, Kim AC, Lee KA, Yoon JH, Ryu JH, Lee WJ. Drosophila microbiome modulates host developmental and metabolic homeostasis via insulin signaling. Science. 2011;334(6056):670-4

64. Storelli G, Defaye A, Erkosar B, Hols P, Royet J, Leulier F. Lactobacillus plantarum promotes Drosophila systemic growth by modulating hormonal signals through TOR-dependent nutrient sensing. Cell Metab. 2011;14(3):403-14.

65. Erkosar B, Storelli G, Defaye A, Leulier F. Host-intestinal microbiota mutualism: "learning on the fly". Cell Host Microbe. 2013;13(1):8-14.

66. Ridley EV, Wong AC, Westmiller S, Douglas AE. Impact of the resident microbiota on the nutritional phenotype of Drosophila melanogaster. PLoS ONE. 2012;7(5):e36765.

67. Spor A, Koren O, Ley R. Unravelling the effects of the environment and host genotype on the gut microbiome. Nat Rev Microbiol. 2011;9(4):279-90

68. Yamada Y, Yukphan P. Genera and species in acetic acid bacteria. Int J Food Microbiol. 2008;125:15-24.

69. Fenn WO, Henning M, Philpott M. Oxygen poisoning in Drosophila. J Gen Physiol. 1967;50(6):1693-707.

70. Biteau B, Karpac J, Supoyo S, DeGennaro M, Lehmann R, Jasper H. Lifespan extension by preserving proliferative homeostasis in Drosophila. PLoS Genet. 2010;6:e1001159.

\section{Submit your next manuscript to BioMed Central and we will help you at every step:}

- We accept pre-submission inquiries

- Our selector tool helps you to find the most relevant journal

- We provide round the clock customer support

- Convenient online submission

- Thorough peer review

- Inclusion in PubMed and all major indexing services

- Maximum visibility for your research

Submit your manuscript at www.biomedcentral.com/submit
O Biomed Central 